\title{
Recombination technologies for enhanced transgene stability in bioengineered insects
}

\author{
Marc F. Schetelig • Frank Götschel • \\ Ivana Viktorinová • Alfred M. Handler • \\ Ernst A. Wimmer
}

Received: 20 April 2010/Accepted: 27 August 2010/Published online: 16 September 2010

(C) The Author(s) 2010. This article is published with open access at Springerlink.com

\begin{abstract}
Transposon-based vectors currently provide the most suitable gene transfer systems for insect germ-line transformation and are used for molecular improvement of the Sterile Insect Technique. However, the long time stability of genome-integrated transposon constructs depends on the absence of transposase activity that could remobilize the transposon-embedded transgenes. To achieve transgene stability transposon vectors are usually non-autonomous, lacking a functional transposase gene, and chosen so that endogenous or related transposon activities are not present in the host. Nevertheless, the non-autonomous transposonembedded transgenes could become unstable by the unintended presence of a mobilizing transposase that may have been undetected or subsequently entered the host species by horizontal gene transfer. Since the field release of
\end{abstract}

M. F. Schetelig - A. M. Handler

USDA/ARS, Center for Medical, Agricultural and Veterinary

Entomology, 1700 SW 23rd Drive, Gainesville, FL 32608, USA

F. Götschel

Division of Molecular Genome Analysis, German Cancer

Research Center (DKFZ), Im Neuenheimer Feld 580,

Heidelberg, Germany

I. Viktorinová

Max Planck Institute of Molecular Cell Biology and Genetics,

Pfotenhauerstr. 108, 01307 Dresden, Germany

E. A. Wimmer $(\bowtie)$

Department of Developmental Biology,

Georg-August-University Göttingen, GZMB,

Ernst-Caspari-Haus, Johann-Friedrich Blumenbach Institute

of Zoology and Anthropology, Justus-von-Liebig-Weg 11,

37077 Göttingen, Germany

e-mail: ewimmer@gwdg.de transgenic insects will present environmental concerns relating to large populations and high mobility, it will be important to ensure that transgene constructs are stably integrated for maintaining strain integrity and eliminating the possibility for unintentional transfer into the genome of another organism. Here we review efficient methods to delete or rearrange terminal repeat sequences of transposons necessary for their mobility, subsequent to their initial genomic integration. These procedures should prevent transposase-mediated remobilization of the transgenes, ensuring their genomic stability.

Keywords $F R T / F l p \cdot p h i C 31$ integrase $\cdot$ piggyBac $\cdot$ Sitespecific recombination $\cdot$ Sterile Insect Technique $\cdot$ Insect pest management

\section{Introduction}

Reliable and stable transformation systems are now available and should be applied to insect pest management programs in the near future: for example, to control insects that heavily damage agriculture and forestry or transmit deadly diseases to animals and humans. Genetic control based on the Sterile Insect Technique (SIT) (Dyck et al. 2005) uses the release of mass-reared, sterile male insects to cause infertile matings that reduce the pest population level (Knipling 1955; Krafsur 1998; Klassen and Curtis 2005). SIT is considered as an environmental-friendly alternative to insecticides for insect species that can be mass reared in artificial settings. SIT has been successfully employed in area-wide approaches to suppress or eradicate pest insects such as the pink bollworm Pectinophora gossypiella in California (Henneberry 2007), the tsetse fly Glossina austeni in Zanzibar (Vreysen 2001), the new 
world screwworm Cochliomyia hominivorax in North and Central America (Wyss 2000), and several tephritid fruit fly species in various regions of the world (Klassen and Curtis 2005).

At least three different types of traits can be engineered and introduced into insect strains to improve their use in the SIT (Robinson and Franz 2000). First, a female-specific lethality system could allow for efficient genetic sexing and the subsequent male-only release; second, a system that causes lethality after transmission to progeny could replace the usual male sterilization by irradiation procedure; and third, marker systems could enable discrimination of released and naturally occurring insects. However, the introduction of these traits requires the heritable germ-line transformation of the target species. This is based on random integration of transgenic DNA into the genome of the target species using transposonbased vectors. Position effects and insertional mutagenesis by the introduced DNA can have several side effects like up- or down-regulation of expression of the introduced system, disrupting the gene structure of the host and a reduced fitness of the transgenic strain. In addition, since transposable elements (TE) are used for gene transfer, the systems bring along the potential risk of transgene instability for the most commonly used vectors. Here we review the most recently developed approaches that ensure post-integrational stabilization of transgenes based on broad-range transposon vectors.

The use of TEs for germ-line transformation is a common method in drosophilid and many non-drosophilid arthropods. The most promising transposons currently available for modification of insect genomes are the $h A T$ family element Hermes originally isolated from Musca domestica (Warren et al. 1994; Subramanian et al. 2009), the Tc1/mariner element Mosl isolated from D. mauritiana (Medhora et al. 1988), the Tcl/mariner element Minos isolated from D. hydei (Franz and Savakis 1991), and the TTAA-specific element piggyBac derived from Trichoplusia ni (Cary et al. 1989), which have been used to successfully transform different insect orders (Berghammer et al. 1999; Handler and James 2000; Atkinson et al. 2001; Handler 2001; Horn et al. 2002; Pavlopoulos et al. 2007).

Functional autonomous transposons typically consist of terminal sequences, including inverted repeat sequences at the ends (in addition to sub-terminal inverted repeat sequences in some elements) that surround a coding region for what is generically referred to as 'transposase'. It is the transposase that binds to the terminal sequences of the TE to catalyze the cut-and-paste process necessary for transposition (Rubin and Spradling 1982). In developing the first transposon-based vector system for insects, Rubin and Spradling (1982) showed that a non-autonomous vector plasmid could be constructed having the transposon terminal sequences surrounding other genes of interest, including a transformation marker gene. If the transposase gene within the vector was deleted or disrupted, transposase could be provided in trans by a helper plasmid having a functional transposase gene, but lacking one or both terminal sequences, thereby preventing the genomic integration of its own. Once the helper facilitates integration of the transposon-embedded transgene in the embryonic germ-line, its loss after cell division should, generally, result in transgene stability. However, the genomic presence of the same or related transposon to that used for integrating the transgene may have influence on the transgene stability.

The presence of similar elements might have a suppressive effect on transposition, as demonstrated by $P$ cytotype repression of the Drosophila $P$ element (Misra and Rio 1990). In this case, transformants would be rarely created and would be of limited concern. Of more concern is the potential for transgene instability due to remobilization by a related transposon having a functional transposase (Sundararajan et al. 1999). For example, studies on $h A T$ elements have shown that a non-autonomous hobo element from Drosophila melanogaster can be cross-mobilized in Musca by the Hermes element, having a similar but not identical transposase (O'Brochta et al. 1994). Moreover, there is evidence for cross-mobilization of related TEs in Drosophila virilis (Evgen'ev et al. 1997). In this species hybrid dysgenesis appears to be due to the retrotransposon Penelope, though expression of this element also seems to mobilize the retrotransposon element Ulysses, and the Tc1/mariner-like element Paris. However, the presence of related TEs does not necessarily lead to the instability of transgenic lines, since most elements are defective lacking functional transposase (Handler and McCombs 2000). For some elements, particular host strains fail to support subsequent remobilization in somatic and/or germ-line cell types. For example, unlike D. melanogaster, in Aedes aegypti there was no evidence for piggyBac re-mobilization after functional piggyBac transposase was supplied (Sethuraman et al. 2007). Thus far, only a limited number and type of elements have been studied in terms of potential positive and negative influences on remobilization. In laboratory "small scale" experiments, cross-mobilization is not an issue. But considering a substitution of irradiated insects for transgenic ones in large-scale field releases of mass reared insects, as used in conventional SIT programs, a major concern for these programs is the stability of transgenic insects during mass rearing maintenance and in the field (Handler 2004). Small-scale experiments and predictions about these transposon-embedded transgenes are not sufficient to safeguard this technology for pest control programs. To date no large-scale experiments have been designed to address these issues-neither for transgenic agricultural pests nor in drosophilids. 
Transgene instability can result, in different ways, in the loss of strain attributes important to the program and result in reduced effectiveness of the biocontrol program (Handler 2004). The development of non-autonomous as well as remobilization-defective transposon insertions is therefore important for the safety of SIT programs when taking transgenic improvements of pest management programs from the lab to the field (Wimmer 2005a, b). The general concern of instability mediated by homologous elements may be addressed by their identification using low stringency Southern hybridization or PCR, (Lohe et al. 1995a, b; Zimowska and Handler 2006; Bonizzoni et al. 2007; Handler et al. 2008) though cross-mobilizing elements having limited sequence similarity could be missed. Existence of such elements could be more definitively determined by highly sensitive in vivo excision or plasmidto-plasmid transposition assays performed in host embryos, where non-autonomous transposon mobility can be determined in the absence of a provided source of transposase (Atkinson et al. 1993; O'Brochta et al. 1994; Sarkar et al. 1997). These assays should ameliorate the major concerns for potential non-autonomous transposon mobilization by a related endogenous element (Ribeiro and Kidwell 1994; Fraser et al. 1995).

\section{Transgene stabilization in Drosophila melanogaster}

Stabilization methods for transgenes have been developed for the model organism $D$. melanogaster in three different ways (Handler et al. 2004; Horn and Handler 2005; Frank Götschel, Ivana Viktorinová, Ernst A. Wimmer, unpublished). First, a vector was created having duplicated 5' terminal piggyBac sequences flanking a transformation marker 1 and an additional $3^{\prime}$ terminal repeat, which flanks, together with the internal $5^{\prime}$ repeat a second clearly distinguishable transformation marker 2 (Fig. 1a). This construction was integrated into Drosophila by piggyBacmediated germline transformation. Flies expressing both markers were isolated and mated to a piggyBac jumpstarter line (having a chromosomal source of transposase). Progeny showing only marker 1 could be isolated and were verified as having only one $5^{\prime}$ terminal repeat from the vector sequence, indicating loss of the $3^{\prime}$ and internal $5^{\prime}$ terminal sequences, along with marker 2 . Stability of the remaining transgene was tested by crossing the line to the piggyBac jumpstarter line and screening their progeny for marker 1 flies. About 3,500 flies were screened that all maintained marker 1 , which supports the conclusion that the non-excised 5'pBac-marker 1 transgenic sequence was stabilized. The major advantage of this transformation system is the possibility to physically delete transposon DNA that is required for transposition following the germ- line transformation process. In this way, transposasemediated mobilization or cross-mobilization of the genesof-interest are excluded mechanistically. In contrast to conventional germ-line transformation technology, the system provides enhanced stability to the transgene insertion as tested in the laboratory experiments. Furthermore, most of the DNA sequences required for the modification are removed from the genome after the final experimental step. A limitation of this system might be that increasing construct size results in decreased integration frequency, thus limiting the number or size of desired transgenes (Handler et al. 2004).

Second, a system was developed in Drosophila that could be beneficial for the applied use of transgenic organisms (once transferred to each particular species) as producers of pharmaceutically relevant proteins or as pest population control agents. To target and modify initially introduced transgenic systems further, site-specific integration sites can be introduced alongside. Site-specific systems frequently used are FRT/FLP, CRE/lox, and the attP-attB/phiC31 (Wimmer 2005b). The system includes the post-integration stabilization method described above, but after providing for precise cassette replacement comprising solely genes of interest (Fig. 1b; Horn and Handler 2005). The method describes transgene targeting to predefined chromosomal sites in Drosophila by using a transposon vector that, once integrated in the germ line, acts as a recombinational acceptor site for donor vectors using the yeast-derived FLP recombinase/FRT recombination sites system. To make recombinational insertions irreversible, a recombinasemediated cassette exchange (RMCE) strategy was used with heterospecific FRT sequences allowing double recombinations, with the use of the homing sequence linotte for enhanced donor-target pairing. Site-specific FRT/FLP recombinants were screened by interconvertible fluorescence marker phenotypes based on the recombinational exchange of the fluorescent protein coding sequence driven by a specific promoter (Fig. 1b; P2), which yielded in targeted insertions at a frequency of about $23 \%$. The RMCE system provides for repetitive integrations into the same locus, allowing comparative analysis of true transgenic alleles. In addition, this method was used to stabilize a targeted transgene by the post-integration excision of putatively mobile piggyBac transposon sequences as described above (Handler et al. 2004).

Third, re-arrangeable gene transfer systems have been developed by also making use of the FRT/FLP system (Frank Götschel, Ivana Viktorinová, Ernst A. Wimmer, unpublished), but for intra-chromosomal recombinations that were previously shown possible in Drosophila (Golic and Golic 1996). Hermes and piggyBac transposon vectors that carry single FRT sites were inserted independently into a genome. The two vectors contained distinguishable 
A Germline transformation; select M1 and M2 marked flies

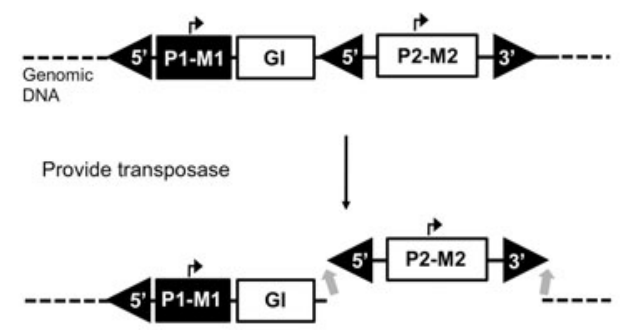

Select progeny marked

with M1, lacking M2

, $\stackrel{\longrightarrow}{P 1-M 1}$

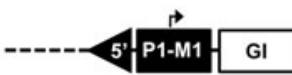

C

Independent germline transformation: select M1 or M2 marked flies

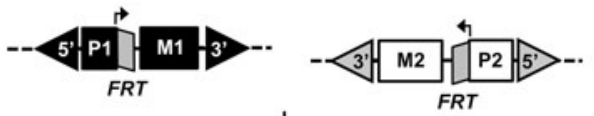

Recombine M1 and M2

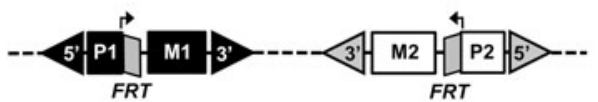

Provide flipase
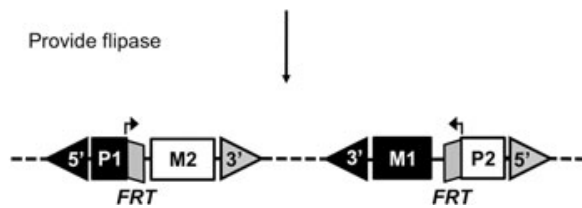

Fig. 1 Stabilization mechanisms for D. melanogaster. Relative positions of fluorescent markers (M1, M2, M3), the promoters (P1, P2), a gene of interest (GI), FRT sites (FRT3, FRT), the homing sequence linotte (HS), and the $5^{\prime} / 3^{\prime}$ transposable ends (triangles) are shown. a After partial remobilization via piggyBac transposase, stabilized flies contain only one $5^{\prime}$ piggyBac end (adapted from Handler et al. 2004). b After RMCE via FLP and heterospecific FRTs as well as subsequent partial remobilization via piggyBac transposase, stabilized flies contain only one $3^{\prime}$ piggyBac end (adapted from Horn

fluorescent protein-based transformation markers driven by different promoters, with the FRTs placed in the $5^{\prime}$ UTR between the respective promoters and the ORF of the markers (Fig. 1c, d). The insertion sites of these transgene constructions were characterized and suitable lines were recombined to produce individuals with both vectors linked on the same chromosome, having their FRT sites in opposite orientation (Fig. 1c, d). After FLP-mediated recombination between two independent $F R T$ sites in inverse orientation on the same chromosome, inversions were generated that could be detected by the fluorescent marker switch due to promoter exchange. The inversion in this case destroys the regular alignment of both the piggyBac as well as the Hermes terminal inverted repeats and
B
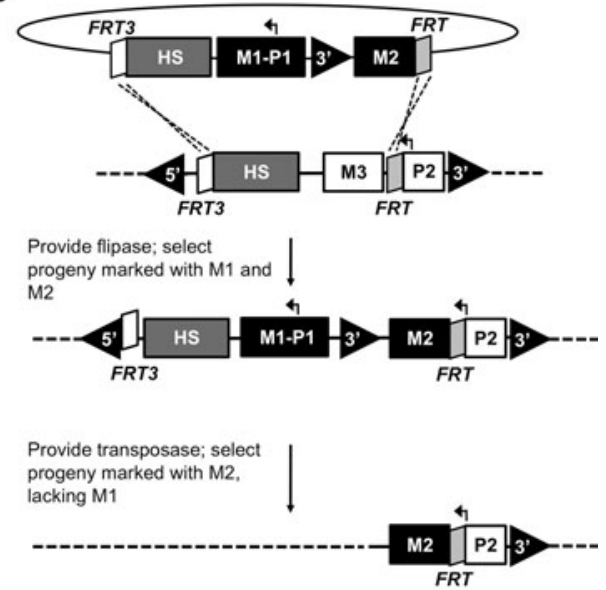

D

Independent germline transformation; select M1 or M2 marked flies
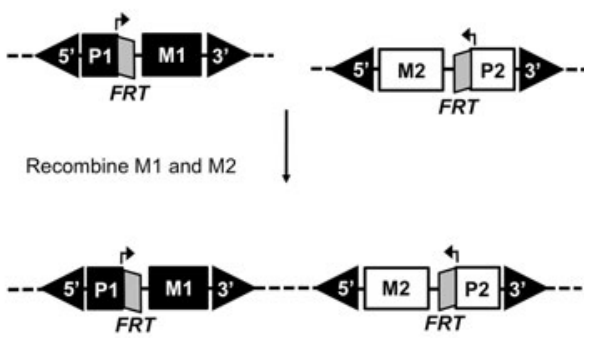

Provide flipase

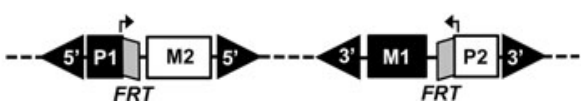

and Handler 2005). c 5'/3' piggyBac (black triangles) and Hermes (gray triangles) transposon ends are shown. FRT/FLP-induced inversion generates two immobile Hermes/piggyBac-hybrid insertions containing one Hermes and one piggyBac end. d All $5^{\prime}$ and $3^{\prime}$ transposable ends are piggyBac ends. After FRT/FLP-induced inversion, the two piggyBac insertions contain either only $5^{\prime}$ or only $3^{\prime}$ ends that makes each of them immobile. Please note: because of the genomic distance (dashed line) the two insertions cannot transpose together either

the rearranged piggyBac/Hermes hybrid transgenic DNA resulted in non-remobilizable transgene insertions with respect to the presence of both corresponding transposases (Fig. 1c). Such inversions can also be generated by using only one type of TEs. By using vectors with different markers having the inserted FRTs in opposite orientation relative to the transposon terminal sequences, recombination-induced inversions generated transgenic DNA insertions with either two $3^{\prime}$ or two $5^{\prime}$ ends that are each refractory to transposase-mediated remobilization and because of the genomic distance of the insertion sites cannot be deleted or transposed together (Fig. 1d). For every line, transgene stability was tested and verified by screening 3,000-4,000 flies. 
These genomic targeting and stabilization strategies described for Drosophila should be applicable to other insects, specifically for the goals of optimizing heterologous protein expression and enhancing the ecological safety of transgenic strains intended for release in biocontrol programs such as SIT programs for the Mediterranean fruit fly and other pest species.

\section{Transgene stabilization in Ceratitis capitata}

Transgene stabilization for the Mediterranean fruit fly (medfly), Ceratitis capitata, has been achieved by a similar method to the one described by Handler et al. (2004). The primary method of deleting transposable ends has been maintained, but instead of deleting one piggyBac end, Dafa' alla et al. (2006) created a system which deletes both $5^{\prime}$ and $3^{\prime}$ ends (Fig. 2a). The initial transformation was performed with a plasmid carrying two pairs of $5^{\prime} / 3^{\prime}$ terminal inverted piggyBac sequences, with independent fluorescent markers within each pair (M1 and M3, respectively; Fig. 2a). In addition these two pairs flank a central region carrying another fluorescent marker (M2). After integration of all four terminal repeats by germ-line transformation (presumably using the external $5^{\prime}$ and $3^{\prime}$ termini) as verified by expression of the three fluorescent transformation markers, the pairs of inverted terminal repeats were remobilized by re-exposure to transposase. After a maximum of three rounds of re-exposure, both pairs were deleted, leaving only the central region with its marker M2, with none of transposon terminal repeat sequences remaining. In terms of applicability to medfly

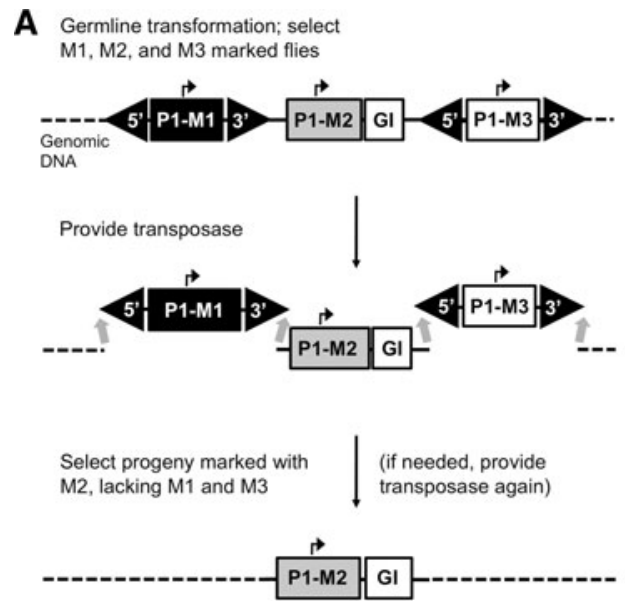

Fig. 2 Stabilization mechanisms for $C$. capitata. Relative positions of fluorescent markers (M1, M2, M3) and their promoters (all P1), the gene of interest (GI), attachment sites for site-specific integration $(a t t P, a t t B, a t t R, a t t L)$, and $5^{\prime} / 3^{\prime}$ piggyBac ends are shown. a After remobilization via piggyBac transposase, flies are piggyBac-end free genomics this system is subject to the same limitations as the simpler stabilization method of Handler et al. (2004). Since the initially integrated constructs require additional piggyBac ends and additional fluorescent markers together with their promoters, this increased construct size is likely to lead to a decreased integration frequency. As an advantage the group reported that no terminal repeat is left in the genome. This system has already been used to generate a transgenic medfly sexing strain (Morrison et al. 2009), though stability tests for this system have yet to be reported.

In addition to the described systems, transgene stability combined with targeting by site-specific recombination was developed for medfly by Schetelig et al. (2009b), who described an efficient method for the modification of previously inserted transgenes by the use of the site-specific integration system from phage phiC31. First, suitable transgenic strains with randomly integrated attP landing sites embedded in transposon-inserted transgenic DNA were identified by molecular and functional characterization. These strains contained a construct with one $5^{\prime}$ and one $3^{\prime}$ piggyBac terminal repeat flanking a fluorescent marker (M1) and an attP landing site for site-specific recombination (Fig. 2b). Second, donor plasmids containing an $a t t B$ site, an additional marker (M2), and an additional $3^{\prime}$ transposon end were integrated into the attP site by phiC31 integrase-mediated recombination (Fig. 2b). This created strains with one $5^{\prime}$ and two tandem $3^{\prime}$ terminal repeats along both markers M1 and M2 (Fig. 2b). Transposase-encoding 'jumpstarter' strains were created and mated to these transgenic strains resulting in the postintegrational excision of transposon ends, which left stably

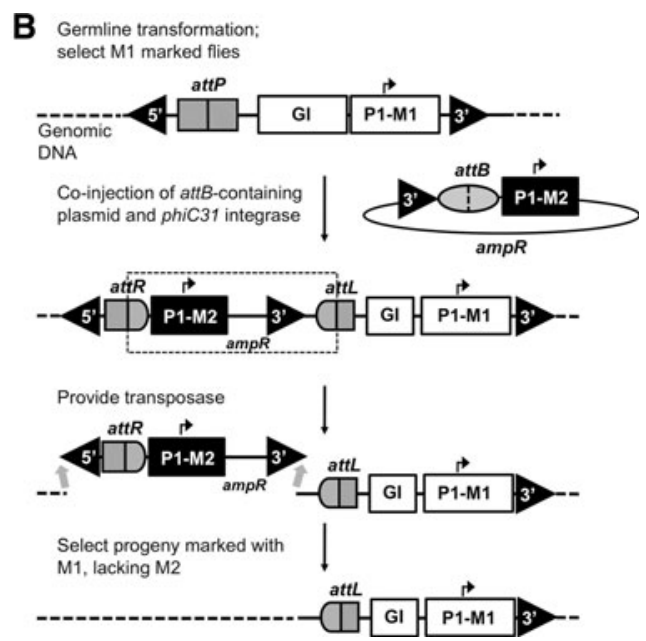

(adapted from Dafa'alla et al. 2006). b After site-specific recombination via phiC31 integrase and subsequent partial remobilization via piggyBac transposase, stabilized flies contain a transgene construct with only one $3^{\prime}$ piggyBac end (adapted from Schetelig et al. 2009b) 
integrated transgene insertions that were identified by the presence of M1 only and could not be further remobilized. This was tested by large-scale laboratory stability tests of about 35,000 flies for two independent strains, where no remobilization events were detected indicating stability. As a control, their respective original versions were also crossed to the jumpstarter strains, resulting in remobilizations and excisions. This three-step integration and stabilization system should allow the combination of several transgene-encoded advantageous traits at evaluated genomic positions in medfly to generate optimized strains for population control, with transgene stabilization minimizing environmental concerns. Advantages of this system are that once a random insertion into a specific genomic locus has been identified as particularly useful with respect to transgene expression, the ability to make the insertion homozygous, and the lack of fitness costs, it can be used for further modifications by site-specific integration. In particular, the attP system has been proven to integrate DNA up to $140 \mathrm{~kb}$ in a one-way fashion (Venken et al. 2006, 2009). This could increase the integration efficiency of large constructs.

\section{Discussion}

The successful application of site-specific recombination technologies should confer great flexibility to insect transgenesis strategies. RMCE makes possible the targeted integration of DNA cassettes into a specific genomic DNA locus, enabling the combination of different transgenic systems in agricultural pest species. These positions are predefined by the integration of the RMCE acceptor plasmid and can be characterized prior to a targeting experiment. Using site-specific systems, researchers should be able to create transposon-free cassette exchange systems for replacement of original transgenes with any desired transgenic construct and to more efficiently combine different systems. Different transgenes can be placed precisely at the same genomic locus, eliminating genomic positional effects and to comparatively study the biological effects of different transgenes. This should greatly facilitate the detailed study of insect pest regulatory elements such as enhancers, silencers, and insulators or the function of insect pest protein variants by the use of expression systems (Wimmer 2005b). It will also enable the creation of transgenic strains carrying diverse transgenes in tandem, which will be coinherited by their progeny. For example, a combination of a conditional embryonic lethality (Schetelig et al. 2009a), a sperm marking (Scolari et al. 2008), and a sexing system (Caceres 2002; Fu et al. 2007) could be put into effect in medfly with already existing and evaluated strains.
However, the different systems available to integrate, exchange, or mobilize DNA differ in their ability to handle large DNA fragments. Transposition efficiency using transposons often decreases when reaching a construct size of 18-25 kb. So using solely transposons for inserting large DNA systems might not be feasible. Instead, reliable and well-documented transposons can be used to integrate the landing sites for site-specific recombination systems, which enable the exchange and integration of larger DNA fragments (Venken et al. 2009). Deleting transposon ends will then further increase the stability of such strains. Having all these systems in one genomic position, proven to be noncompromising and effective, should improve application processes including sterilization, sexing, and monitoring of ongoing SIT programs. We would expect that the generated stabilized lines show the same fitness as their non-stabilized predecessors, since the immobilization procedure only removes part of the transposon construct that did not exist in the wild type host. Nevertheless, the fitness of stabilized lines should be verified by large-scale field cage competition assays before an eventual field application.

Transgenic technology relying on transposon-based vectors has proven to be a powerful tool for the genetic manipulation of insect genomes, which can be tested and applied to the improvement of current environmentfriendly pest control methods (Wimmer 2003). However, a fundamental requirement for a safe release of genetically modified insects into the wild is the development of systems inert to any potential mobilizing transposase source present in the environment. Therefore the development of non-autonomous transposons whose ends can be deleted or rearranged post-integration to cause effective immobilization of the inserted transgene is essential to ultimately taking transgenic improvements of pest management programs from the lab to the field (Wimmer 2005a, b). The development of such systems for medfly as a major pest species has been achieved (Dafa'alla et al. 2006; Schetelig et al. 2009b) and large-scale laboratory stability tests have proven that these modified insertions are inert to the presence of the piggyBac transposase (Schetelig et al. 2009b). This represents an important step forward towards the safe field use of transgenic insects.

For the initial evaluations of the environmental impact of transgenic insects, SIT programs, with their high quality control during sterile insect production, should minimize any potential ecological concerns for the release of transgenic insects (Robinson and Franz 2000; Robinson and Hendrichs 2005). The sterility of the released insects will serve as a biological safety mechanism that impedes vertical transmission of the transgenes, which will be eliminated from the ecosystem concurrent with cessation of the SIT program. 
Acknowledgments Cited research from our labs has been supported by the Robert Bosch Foundation (EAW) within the program 'International Research into the Development of Sustainable Agriculture and Forestry', the Fonds der Chemischen Industrie (EAW), the Bundesministerium für Bildung und Forschung (EAW), the European Molecular Biology Organization Young Investigator Programme (EAW), and United States Department of Agriculture-Agricultural and Food Research Initiative (AMH). For many of the ideas presented here, we are indebted to informative discussions with expert colleagues, especially at the International Atomic Energy Agency funded meetings of the Coordinated Research Projects "The Use of Molecular Tools to Improve the Effectiveness of SIT"' and "Development and Evaluation of Improved Strains of Insect Pests for SIT".

Open Access This article is distributed under the terms of the Creative Commons Attribution Noncommercial License which permits any noncommercial use, distribution, and reproduction in any medium, provided the original author(s) and source are credited.

\section{References}

Atkinson PW, Warren WD, O'Brochta DA (1993) The hobo transposable element of Drosophila can be cross-mobilized in houseflies and excises like the Ac element of maize. Proc Natl Acad Sci USA 90:9693-9697

Atkinson PW, Pinkerton AC, O'Brochta DA (2001) Genetic transformation systems in insects. Annu Rev Entomol 46:317-346

Berghammer AJ, Klingler M, Wimmer EA (1999) A universal marker for transgenic insects. Nature 402:370-371

Bonizzoni M, Gomulski LM, Malacrida AR, Capy P, Gasperi G (2007) Highly similar piggyBac transposase-like sequences from various Bactrocera (Diptera, Tephritidae) species. Insect Mol Biol 16:645-650

Caceres C (2002) Mass rearing of temperature sensitive genetic sexing strains in the Mediterranean fruit fly (Ceratitis capitata). Genetica 116:107-116

Cary LC, Goebel M, Corsaro BG, Wang HG, Rosen E, Fraser MJ (1989) Transposon mutagenesis of baculoviruses: analysis of Trichoplusia ni transposon IFP2 insertions within the FP-locus of nuclear polyhedrosis viruses. Virology 172:156-169

Dafa'alla TH, Condon GC, Condon KC, Phillips CE, Morrison NI, Jin L, Epton MJ, Fu G, Alphey L (2006) Transposon-free insertions for insect genetic engineering. Nat Biotechnol 24:820-821

Dyck VA, Hendrichs J, Robinson AS (2005) Sterile insect techniqueprinciples and practice in area-wide integrated pest management. Springer, Dordrecht

Evgen'ev MB, Zelentsova H, Shostak N, Kozitsina M, Barskyi V, Lankenau DH, Corces VG (1997) Penelope, a new family of transposable elements and its possible role in hybrid dysgenesis in Drosophila virilis. Proc Natl Acad Sci USA 94:196-201

Franz G, Savakis C (1991) Minos, a new transposable element from Drosophila hydei, is a member of the Tcl-like family of transposons. Nucleic Acids Res 19:6646

Fraser MJ, Cary L, Boonvisudhi K, Wang HG (1995) Assay for movement of Lepidopteran transposon IFP2 in insect cells using a baculovirus genome as a target DNA. Virology 211:397-407

Fu G, Condon KC, Epton MJ, Gong P, Jin L, Condon GC, Morrison NI, Dafa'alla TH, Alphey L (2007) Female-specific insect lethality engineered using alternative splicing. Nat Biotechnol 25:353-357

Golic KG, Golic MM (1996) Engineering the Drosophila genome: chromosome rearrangements by design. Genetics 144:1693-1711
Handler AM (2001) A current perspective on insect gene transformation. Insect Biochem Mol Biol 31:111-128

Handler AM (2004) Understanding and improving transgene stability and expression in insects for SIT and conditional lethal release programs. Insect Biochem Mol Biol 34:121-130

Handler AM, James AA (2000) Insect transgenesis: methods and applications. CRC Press LLC, Boca Raton

Handler AM, McCombs SD (2000) The piggyBac transposon mediates germ-line transformation in the Oriental fruit fly and closely related elements exist in its genome. Insect Mol Biol 9:605-612

Handler AM, Zimowska GJ, Horn C (2004) Post-integration stabilization of a transposon vector by terminal sequence deletion in Drosophila melanogaster. Nat Biotechnol 22:1150-1154

Handler AM, Zimowska GJ, Armstrong KF (2008) Highly similar piggyBac elements in Bactrocera that share a common lineage with elements in noctuid moths. Insect Mol Biol 17:387-393

Henneberry $T$ (2007) Integrated systems for control of the pink bollworm Pectinophora Gossypiella in cotton. In: Vreysen MJB, Robinson AS, Hendrichs J (eds) Area-wide control of insect pests: from research to field implementation. Springer, Dordrecht, pp 567-579

Horn C, Handler AM (2005) Site-specific genomic targeting in Drosophila. Proc Natl Acad Sci USA 102:12483-12488

Horn C, Schmid BG, Pogoda FS, Wimmer EA (2002) Fluorescent transformation markers for insect transgenesis. Insect Biochem Mol Biol 32:1221-1235

Klassen W, Curtis CF (2005) History of the sterile insect technique. In: Dyck VA, Hendrichs J, Robinson AS (eds) Sterile insect technique. Principles and practice in area-wide integrated pest management. Springer, Dordrecht, pp 3-36

Knipling EF (1955) Possibilities of insect control or eradication through the use of sexually sterile males. J Econ Entomol 48:459-462

Krafsur ES (1998) Sterile insect technique for suppressing and eradicating insect population: 55 years and counting. J Agric Entomol 15:303-317

Lohe AR, Lidholm DA, Hartl DL (1995a) Genotypic effects, maternal effects and grand-maternal effects of immobilized derivatives of the transposable element mariner. Genetics 140:183-192

Lohe AR, Moriyama EN, Lidholm DA, Hartl DL (1995b) Horizontal transmission, vertical inactivation, and stochastic loss of mariner-like transposable elements. Mol Biol Evol 12:62-72

Medhora MM, MacPeek AH, Hartl DL (1988) Excision of the Drosophila transposable element mariner: identification and characterization of the Mos factor. EMBO J 7:2185-2189

Misra S, Rio DC (1990) Cytotype control of Drosophila P element transposition: the $66 \mathrm{kd}$ protein is a repressor of transposase activity. Cell 62:269-284

Morrison NI, Segura DF, Stainton KC, Fu G, Donnelly CA, Alphey LS (2009) Sexual competitiveness of a transgenic sexing strain of the Mediterranean fruit fly, Ceratitis capitata. Entomol Exp Appl 133:146-153

O'Brochta DA, Warren WD, Saville KJ, Atkinson PW (1994) Interplasmid transposition of Drosophila hobo elements in nondrosophilid insects. Mol Gen Genet 244:9-14

Pavlopoulos A, Oehler S, Kapetanaki MG, Savakis C (2007) The DNA transposon Minos as a tool for transgenesis and functional genomic analysis in vertebrates and invertebrates. Genome Biol 8(Suppl 1):S2

Ribeiro JM, Kidwell MG (1994) Transposable elements as population drive mechanisms: specification of critical parameter values. J Med Entomol 31:10-16

Robinson AS, Franz G (2000) The application of transgenic insect technology in the sterile insect technique. In: Handler AM, 
James AA (eds) Insect transgenesis: methods and applications. CRC Press LLC, Florida, pp 307-319

Robinson AS, Hendrichs J (2005) Prospects for the future development and application of the sterile insect technique. In: Dyck VA, Hendrichs J, Robinson AS (eds) Sterile insect techniqueprinciples and practice in area-wide integrated pest management. Springer, Dordrecht, pp 727-760

Rubin GM, Spradling AC (1982) Genetic transformation of Drosophila with transposable element vectors. Science 218:348-353

Sarkar A, Coates CJ, Whyard S, Willhoeft U, Atkinson PW, O'Brochta DA (1997) The Hermes element from Musca domestica can transpose in four families of cyclorrhaphan flies. Genetica 99:15-29

Schetelig MF, Caceres C, Zacharopoulou A, Franz G, Wimmer EA (2009a) Conditional embryonic lethality to improve the sterile insect technique in Ceratitis capitata (Diptera: Tephritidae). BMC Biol 7:4

Schetelig MF, Scolari F, Handler AM, Kittelmann S, Gasperi G, Wimmer EA (2009b) Site-specific recombination for the modification of transgenic strains of the Mediterranean fruit fly Ceratitis capitata. Proc Natl Acad Sci USA 106:18171-18176

Scolari F, Schetelig MF, Bertin S, Malacrida AR, Gasperi G, Wimmer EA (2008) Fluorescent sperm marking to improve the fight against the pest insect Ceratitis capitata (Wiedemann; Diptera: Tephritidae). N Biotechnol 25:76-84

Sethuraman N, Fraser MJ Jr, Eggleston P, O'Brochta DA (2007) Postintegration stability of piggyBac in Aedes aegypti. Insect Biochem Mol Biol 37:941-951

Subramanian RA, Cathcart LA, Krafsur ES, Atkinson PW, O'Brochta DA (2009) Hermes transposon distribution and structure in Musca domestica. J Hered 100:473-480
Sundararajan P, Atkinson PW, O'Brochta DA (1999) Transposable element interactions in insects: crossmobilization of hobo and Hermes. Insect Mol Biol 8:359-368

Venken KJ, He Y, Hoskins RA, Bellen HJ (2006) P[acman]: a BAC transgenic platform for targeted insertion of large DNA fragments in D. melanogaster. Science 314:1747-1751

Venken KJ, Carlson JW, Schulze KL, Pan H, He Y, Spokony R, Wan $\mathrm{KH}$, Koriabine M, de Jong PJ, White KP et al (2009) Versatile $\mathrm{P}$ [acman] BAC libraries for transgenesis studies in Drosophila melanogaster. Nat Methods 6:431-434

Vreysen MJ (2001) Principles of area-wide integrated tsetse fly control using the sterile insect technique. Med Trop (Mars) 61:397-411

Warren WD, Atkinson PW, O'Brochta DA (1994) The Hermes transposable element from the house fly, Musca domestica, is a short inverted repeat-type element of the hobo, Ac, and Tam3 (hAT) element family. Genet Res 64:87-97

Wimmer EA (2003) Innovations: applications of insect transgenesis. Nat Rev Genet 4:225-232

Wimmer EA (2005a) Eco-friendly insect management. Nat Biotechnol 23:432-433

Wimmer EA (2005b) Insect transgenesis by site-specific recombination. Nat Methods 2:580-582

Wyss JH (2000) Screwworm eradication in the Americas - overview, (ed. K Tan). Pulau Pinang: Penerbit Universiti Sains Malaysia

Zimowska GJ, Handler AM (2006) Highly conserved piggyBac elements in noctuid species of Lepidoptera. Insect Biochem Mol Biol 36:421-428 